\title{
(6) OPEN ACCESS \\ Examining perceptions about IQOS heated tobacco product: consumer studies in Japan and Switzerland
}

\author{
Elizabeth C Hair, ${ }^{1,2}$ Morgane Bennett, ${ }^{1,3}$ Emily Sheen, ${ }^{4}$ Jennifer Cantrell, ${ }^{2,5}$ \\ Jodie Briggs, ${ }^{1}$ Zoe Fenn, ${ }^{4}$ Jeffrey G Willett, ${ }^{1}$ Donna Vallone ${ }^{1,2,5}$
}

\begin{abstract}
${ }^{1}$ Schroeder Institute, Truth Initiative, Washington, DC, USA ${ }^{2}$ Department of Health, Behavior and Society, Bloomberg School of Public Health, Johns Hopkins University, Baltimore, MD, USA ${ }^{3}$ Department of Prevention and Community Health, Milken Institute School of Public Health, The George Washington University, Washington, DC, USA ${ }^{4}$ Flamingo, London, UK ${ }^{5}$ College of Global Public Health, New York University, New York, NY, USA
\end{abstract}

\section{Correspondence to} Dr Elizabeth C Hair, Schroeder Initiative, Truth Initiative, Washington, DC, USA; ehair@truthinitiative.org

Received 16 February 2018 Revised 23 April 2018 Accepted 26 April 2018 Published Online First 15 May 2018

Check for updates

To cite: Hair EC, Bennett M, Sheen $\mathrm{E}$, et al. Tob Control 2018:27:570-s73.

\section{ABSTRACT}

Objective To examine consumer perceptions, attitudes and behaviours regarding the heated tobacco product, IQOS, as well as to document the product's marketing strategies to determine its potential for appealing to youth and young adults.

Method Truth Initiative, in collaboration with Flamingo, collected qualitative data via: (1) expert interviews, (2) semiotic analysis of IQOS packing and marketing materials, and (3) 12 focus groups with adults in Switzerland (ages 19-44 years; June 6-9, 2016) and Japan (ages 20-39 years; June 22-24, 2016) ( $n=68$ for both groups).

Results Expert interviews and IQOS packing and marketing analyses revealed the product is being marketed as a clean, chic and pure product, which resonated very well in Japan given the strong cultural values of order, cleanliness, quality and respect for others. Focus groups results indicated Japanese IQOS users used the product for socialising with non-smokers. Focus group participants in both Japan and Switzerland reported lower levels of satisfaction with the product relative to combustible cigarettes, although many found the product packaging to be appealing. While participants identified several benefits and barriers related to IQOS, few reported any potential health benefits of use compared with combustible tobacco products.

Conclusion IQOS was marketed as a sophisticated, high tech and aspirational product. Because youth and young adults are more interested in such product positioning, this approach raises some concern about youth appeal. This research shows cultural factors appeared to affect the appeal of this messaging, indicating that prevalence and uptake data will likely not be similar from country to country.

\section{INTRODUCTION}

The heated tobacco product (HTP), IQOS, is a battery-powered, pen-like device that delivers nicotine to users by heating tobacco. This device was developed by Phillip Morris International (PMI) and released to the market in 2014. Users operate it by inserting a 'HeatStick', a rod of tobacco that is electrically heated to a high temperature without igniting and combusting like a traditional combustible cigarette. According to PMI, nicotine is delivered to the user through this heating process with a taste similar to a traditional combustible cigarette. ${ }^{1}$ These devices are entering markets at a time when e-cigarette popularity is beginning to wane, and research suggests e-cigarette users are dissatisfied with the lack of rapid nicotine delivery and 'throat-hit' that come from combustible tobacco use. $^{2}$ The use of real tobacco in IQOS may make this new device a more appealing alternative to those looking for a seemingly less harmful alternative to combustible tobacco. Tobacco executives appear to be aware of this opportunity and prepared to take advantage of it, with PMI CEO reportedly stating HTPs are the 'greatest growth opportunity in the years to come, which we believe has the very real potential to transform the industry'.

IQOS is marketed as a clean alternative to cigarettes using online promotional materials that present the device as sophisticated, high tech and providing all the benefits of smoking but less ash and odour. ${ }^{1}$ IQOS products were first test-marketed in Japan and Italy in 2014 and are now being test-marketed in 30 countries around the world, including Switzerland. ${ }^{4}$ Japan, however, remains the only country that has seen a national roll-out of IQOS. A longitudinal online survey of youth and adults in Japan found less than 1\% of respondents had ever used an IQOS device from January to March 2015. However, that percentage doubled in 2016 and reached $3.6 \%$ by February 2017, with an estimated 3 million people in Japan using IQOS. ${ }^{4}$ Analysis of Google search data revealed dramatic increases in searches for HTPs between 2014 and 2017 in Japan, a further indication of the rapid growth in interest and popularity of the products. ${ }^{5}$ Surveys of Italian youth and adults revealed that almost 20\% were aware of IQOS, and 2.3\% reported intentions to try the device in the future. ${ }^{6}$ Another indication of the growing popularity of IQOS is its increasing share of the tobacco product market, reaching over $13 \%$ of the market share of all tobacco products in Japan by October 2017 .

In 2016, PMI filed an application to the US Food and Drug Administration (FDA) for IQOS to be marketed as a modified risk tobacco product in the USA. To date, FDA continues to accept comments to the PMI application. ${ }^{8}$ In January 2018, the FDA Center for Tobacco Products, Tobacco Products Scientific Advisory Committee rejected PMI's claim that IQOS is less harmful than cigarettes. However, the committee did concede that evidence suggests that 'switching completely from cigarettes to the IQOS system significantly reduces your body's exposure to harmful or potentially harmful chemicals'. ${ }^{9}$ Given the ongoing review of PMI's application, and the product's growing popularity and expansion into worldwide markets, ${ }^{10}$ the current study was designed to explore knowledge, attitudes, beliefs and marketing strategies related to IQOS use in Japan and Switzerland. 


\section{METHODS}

Original data were gathered by Truth Initiative, a US-based non-profit public health organisation focused on tobacco prevention, and Flamingo, an insight and brand consultancy firm based in the UK, via expert interviews, product and marketing analyses and focus groups.

\section{Expert interviews}

Expert interviews were conducted with professionals working in youth culture and youth and young adult tobacco and electronic nicotine delivery system use in order to gain insight into how IQOS might translate into markets. Experts were identified via desk research and included an ethnographer specialising in e-cigarettes and young adult smoking and an editor at Vice.com who specialises in youth culture. These experts were selected to ensure both an academic and a cultural lens on smoking and were financially incentivised for their time. Questions asked via a structured discussion guide encouraged experts to explain their understanding of youth smoking culture to unpack the differences between what cigarettes and e-cigarettes represent in culture. Experts were also asked to hypothesise how IQOS might fit into cultures and markets, based on their understanding of tobacco and e-cigarettes. This process provided key information to better understand how IQOS's marketing fits into broader cultural landscapes. Questions covered areas such as, 'what attracts young people to smoking and vaping today?' and 'where do you see the future of smoking - what will keep it relevant for young people?'.

Interviews were audio-recorded and transcribed. Transcripts were analysed by two Flamingo semioticians who specialise in connecting product attributes with broader cultural trends. The two researchers performing the analyses differed in their level of involvement with the project (ie, one was closely involved in the design and implementation of the study, while the other was not) in order check the reliability of study findings. A thematic analysis approach was used to place interview findings into cultural contexts, informed by additional research on youth websites, such as Vice.

\section{Product and marketing analysis}

An analysis of IQOS packaging (font, colour scheme, pack and product shape and claims) and advertisements (claims, colours, font and visuals) was also conducted to document the brand positioning and use of terminology. Field workers visited IQOS stores in Tokyo and Zurich, the majority of which were located within close proximity to train stations to ensure high volume of foot traffic and collected print marketing materials and photographs of point-of-sale marketing. The stores visited represented a convenience sample of IQOS retailers in the two cities.

\section{Focus groups}

Results of the expert interviews and IQOS product and marketing analyses were used to inform the focus group guides. Twelve focus groups (six in each country) were conducted in Japan and Switzerland during June 2016. These were stratified according to age (18-19 years, 20-25 years and 26-44 years in Lausanne and Zurich, Switzerland; 20-24 years, 25-29 years and 30-39 years in Nagoya, Japan) and segmented by attitudes to IQOS. A total of 68 participants from both countries were recruited through social media posts, telephone lists and at retail venues that sold IQOS. The cities of Lausanne and Zurich were selected as they were the first cities in Switzerland where IQOS was available, and Nagoya was selected because it was the location
IQOS was initially launched. Recruitment criteria included age (segmented as described above), smoking habits, attitudes to smoking and willingness to talk freely on the subject in a group setting. Participants were recruited to fit within the following smoking categories: (1) those who had fully converted to IQOS use from cigarettes, (2) dual users of both IQOS and cigarettes, (3) those who had tried and rejected IQOS and (4) those who were aware of IQOS but had never tried it. Focus groups included a set of open-ended questions related to respondents' combustible tobacco smoking habits (eg, 'What occasions do you smoke? Who with? What? Why? Do you smoke other things on different occasions? Why/Why not?') and IQOS perceptions (eg, 'How did you discover it? What were your initial impressions? How has your opinion changed?'), usage (eg, 'What moments do you use IQOS? Any moments where you'd be less likely to use it? Why? Why not?') and behaviours (eg, 'How does it compare using IQOS versus regular cigarettes? Has it changed your routine at all? How do you find it to hold?'). Questions also examined receptivity to IQOS marketing and promotional message themes (eg, 'What (if any) communications have you seen for the product? What stands out in your mind? Why? Have you attended any IQOS events? If so, describe the event to me. How did it make you feel?').

Focus groups were audio-recorded and transcribed. Transcriptions were analysed by Flamingo through the four thematic lenses that resulted from the semiotic packaging analysis and expert interviews: cleanliness, customisation, next generation smoking and sociability (see Results section for more details). The analysis team at Flamingo consisted of three qualitative researchers in the UK (at different levels of closeness to the project to maintain objectivity), two semiotics team members (also at different levels of closeness to the project) and two qualitative research/ semiotics hybrids in Japan (who had conducted the Japanese interviews). Analysis methods included recurring sentiment and attitudinal analysis, through which patterns in participants' emotional reactions to the four themes were analysed across markets, and examination of linguistic themes, through which emotive language used was explored in detail in order to identify additional themes.

\section{RESULTS}

\section{Expert interviews}

The expert interviews helped define and examine the intersection of smoking and youth culture, unearthing insights such as: technology's most important role for young people is as an emotional facilitator, and today's youth are more wedded to technology than any previous generation, across all aspects of their lives. One expert stated, 'My younger siblings grew up with Facebook and Snapchat and they grew up not knowing anything else [...] I feel like they don't understand offline etiquette'. Two key spaces emerged from these expert interview discussions around youth culture: freedom and control-a tension between using technology as freedom of expression, to pursue emotional desires, and set yourself apart, but also to control your body, organise your life and uncover the processes behind the goods they consume. The expert interviews suggested that vaping speaks to the freedom space (rebellion, smoke, 'hackable' nature of the device and no clear rules), while IQOS would likely sit more in the control space (clean lines, official branding, not 'hackable' or flexible in terms of flavour).

\section{Product and marketing analysis results}

Analysis of marketing strategies revealed a comprehensive effort to promote IQOS as a sophisticated and aspirational product in 
both Japan and Switzerland. IQOS promotional efforts centred around presenting the product in a clean, controlled, minimalist environment during invitation-only pop-up events in dedicated spaces. These events introduced the product by employing brand ambassadors to showcase the product and answer questions with free samples. These brand representatives highlighted the sleek, exclusive 'iPhone' style and quality of IQOS products, as well as the benefits of reduced ash and odour. An analysis of the product's marketing and advertising in both countries identified four key message themes: cleanliness, customisation, comparisons with combustible smoking and sociability. The overarching message architecture focused on the concept of modernising traditional smoking by promoting themes of control and freedom from the negative aspects of combustible tobacco smoking. Analysis of the product packaging revealed eight additional themes, specific to the device: clinical purity, a closed system, premium design, sensory invitations, nostalgia for combustible tobacco smoking, stability, familiar technology and normalisation. For example, the product's 'clinical purity' allowed smokers to control offensive factors like smoke and ash, distinguishing the product from combustible cigarettes. At the time of data collection, any potential health benefits associated with IQOS use were not included on the product packaging or marketing materials in either country. Some marketing materials in Switzerland contained the health message, 'This tobacco product can harm your health and is addictive'.

IQOS marketing efforts in both countries were also found to highlight product factors that are similar to traditional combustible cigarettes in an effort to invoke familiarity and nostalgia for smoking. Marketing materials highlight the similarity of the product's taste and behavioural process to combustible cigarettes, the similarity between the size of HeatSticks and combustible cigarettes and the charging mechanism to an old-fashioned cigarette lighter. Additionally, IQOS products are occasionally displayed next to combustible cigarettes in stores.

\section{Focus group results}

Focus group participants in Japan consistently reported IQOS as a clean, chic and pure product, indicating the effectiveness of the marketing strategy. Respondents primarily reported using IQOS when socialising with groups of non-smokers where the use of combustible cigarettes could infringe on smoke-free social situations. One respondent commented, 'Most of my friends have little kids and I started feeling uncomfortable smoking around them. So now I am only using IQOS'. Participants also reported using the product in places where smoking combustible cigarettes may leave an unwanted residue in an area. One participant stated, 'I like smoking IQOS while watching the TV with my family at home. IQOS is the best for smoking in the house because it creates no ash or odour'. Japanese participants also commented on the cumbersome process of using IQOS. For these respondents, taking along the charger and HeatSticks can be bulky and burdensome. Nonetheless, Japanese focus group participants found the packaging of the product to be appealing. Even non-users unimpressed by descriptions of the device were intrigued when presented with the actual product, indicating that the product's sleek appearance blended well with existing tech devices. Younger non-users commented that price could be a potential barrier, but after analysis of the focus group conversations, this in fact served to contribute to the overall cache of the product as luxurious and prestigious.

In Switzerland, the product's promise of freedom was subverted by the realities of using an HTP. Swiss respondents complained about the charging and cleaning of IQOS. Focus group results suggested a view of smoking combustible cigarettes as a tool for self-expression, and it appeared that the IQOS's promotional efforts failed to resonate in this culture. In reference to smoking combustible cigarettes, one young adult participant stated, 'I guess it's the time where you have that sense of freedom, you're doing something the teachers don't want you to do'. Many focus group participants reported initiating smoking combustible tobacco products to impress friends, rebel against authority figures (eg, teachers and parents) or set a trend. Several participants reported that the product did not provide the same level of intensity as smoking combustible cigarettes, and thus, comparisons with combustible cigarettes made by IQOS were not deemed credible. Swiss participants also cited the milder taste and reduced sensory cues as barriers to continued use. One Swiss participant commented, 'There's just something about that after-work drink, I need a proper cigarette with it. Same with coffee; cigarettes just "go" with coffee'.

Focus group participants in both countries identified several benefits of IQOS use, including less throat discomfort, appealing packaging, cleanliness, lack of ash and smoke and more social acceptability. Participants commented on the novelty of the product as both an advantageous 'conversation starter' for some, as well as 'ostentatious' by others. Participants in both locations also identified several barriers to using the device, including a strange or unpleasant taste and smell, unfamiliar appearance, high maintenance and high cost. Some who had previously smoked combustible cigarettes noted that the product was cumbersome because it could not be held like a traditional combustible cigarette. Among participants in both countries, few identified any potential health benefits of IQOS use compared with combustible tobacco products, and many expressed that the product still felt unfamiliar and complicated to use.

\section{DISCUSSION}

Findings from this exploratory study suggest HTPs, like IQOS, may appeal to consumers, particularly within cultures that value cleanliness, exclusivity and high tech appearances. Others who perceive combustible tobacco use as an expression of freedom, and individualism may be deterred by the price of the product, its cumbersome utility, high maintenance and unfamiliar taste, smell and appearance. The consumer research presented here suggests consumer reception of IQOS may differ depending on culture. Similar cultural differences have been observed in the acceptance and use of snus as a harm reduction tool. While evidence suggests snus may be an effective harm reduction method among Swedish smokers, the same has not been found in the USA. ${ }^{11} 12$

Consistent with the current study, PMI's research that was presented in their modified risk tobacco product application to the US FDA suggested there is more interest in IQOS in Asian markets compared with European markets. ${ }^{13}$ This suggests usage patterns and IQOS acceptance are likely to significantly vary from country to country. However, the popularity of e-cigarettes in the USA and the potential for HTPs to become a more appealing alternative to current e-cigarette users highlights the need to further monitor the launch of novel HTPs, like IQOS, in US markets.

Evidence from tobacco industry executives suggests a strong desire and interest in heavily promoting HTPs in order to take advantage of the declining consumer interest in combustible tobacco products and e-cigarettes. ${ }^{2}$ As was historically 
done with combustible cigarette promotions, ${ }^{14}$ the marketing strategies used by PMI for HTPs may seek to capitalise on the products' potential among youth and young adults in the USA-a group for whom combustible cigarette use continues to decline. ${ }^{15}$ Marketing efforts to portray HTPs as sleek, exclusive items akin to iPhones could find success among American teens and young adults, and researchers are already warning of growing interest and potential demand within new markets. ${ }^{516}$

\section{What this paper adds}

- To our knowledge, this is the first study of IQOS conducted independent of a tobacco company to provide a brief overview of the marketing and promotional efforts, as well as consumer responses related to the heated tobacco product (HTP), IQOS, in Japan and Switzerland.

- Tobacco industry executives have indicated significant interest in developing and promoting novel HTPs, like IQOS. Given the probable increasing effort by the industry to promote HTPs, and the pending application for IQOS to be marketed as a modified risk tobacco product in the USA, findings are key in understanding how this product may be promoted and how to counter these efforts for at-risk groups, particularly youth and young adults.

Contributors ECH, JC, JGW and DV designed the study. ES and ZF performed the data collection. MB and JB wrote the paper. All authors contributed to the revising of the paper.

Funding The authors have not declared a specific grant for this research from any funding agency in the public, commercial or not-for-profit sectors.

Competing interests None declared.

Patient consent Not required.

Provenance and peer review Not commissioned; externally peer reviewed.

Open access This is an open access article distributed in accordance with the Creative Commons Attribution Non Commercial (CC BY-NC 4.0) license, which permits others to distribute, remix, adapt, build upon this work non-commercially, and license their derivative works on different terms, provided the original work is properly cited and the use is non-commercial. See: http://creativecommons.org/ licenses/by-nc/4.0/

(C) Article author(s) (or their employer(s) unless otherwise stated in the text of the article) 2018. All rights reserved. No commercial use is permitted unless otherwise expressly granted.

\section{REFERENCES}

1 Phillip Morris International. IQOS: tobacco meets technology. USA: Phillip Morris International.

2 Caputi TL. Industry watch: heat-not-burn tobacco products are about to reach their boiling point. Tob Control 2016;26:609-10.

3 Gara T. Introducing the New USB-Powered Pack of Marlboros. The Wall Street Journal 2014.

4 Tabuchi T, Gallus S, Shinozaki T, et al. Heat-not-burn tobacco product use in Japan: its prevalence, predictors and perceived symptoms from exposure to secondhand heatnot-burn tobacco aerosol. Tob Control 2018;27(e1):e25-e33.

5 Caputi TL, Leas E, Dredze M, et al. They're heating up: Internet search query trends reveal significant public interest in heat-not-burn tobacco products. PLoS One 2017:12:e0185735

6 Liu X, Lugo A, Spizzichino L, et al. Heat-not-burn tobacco products: concerns from the Italian experience. Tob Contro/ 2018. doi: 10.1136/tobaccocontrol-2017-054054. [Epub ahead of print 26 Jan 2018].

7 Trefis. iQOS: set to drive growth for philip morris in the future: Nasdaq, 2017.

8 U.S. Food and Drug Administration. Philip Morris Products S.A. Modified Risk Tobacco Product (MRTP) Applications, 2017.

9 U.S. Food and Drug Administration. Meeting of the tobacco products scientific advisory committee: Center for Tobacco Products, 2018.

10 Herzog B, Kanada P, Lachky J, et al. iQOS in pictures: a visual overview of the global opportunity through 2025. Wells Fargo Securities 2017.

11 Gilljam H, Galanti MR. Role of snus (oral moist snuff) in smoking cessation and smoking reduction in Sweden. Addiction 2003;98:1183-9.

12 Mejia AB, Ling PM, Glantz SA. Quantifying the effects of promoting smokeless tobacco as a harm reduction strategy in the USA. Tob Control 2010;19:297-305.

13 Office of Science Center for Tobacco Products Food and Drug Administration. Meeting of the Tobacco Products Scientific Advisory Committee (TPSAC): Modified Risk Tobacco Product Applications(MRTPAs). MR0000059-MR0000061, Philip Morris Products S.A.

14 U.S. Department of Health Human Services. Preventing tobacco use among youth and young adults: a report of the Surgeon General. Atlanta, GA, 2012.

15 Johnston L, O'Malley PM, Miech RA, et al. Monitoring the future national survey results on drug use: 1975-2013: Overview of key findings on adolescent drug use. Ann Arbor, MI: Institute for Social Research, The University of Michigan, 2014.

16 Halpern-Felsher BMK, Popova L, Kim M, et al. Ling P IQOS likely appeal to youth and young adults and gateway effects with cigarettes. 2018. 